\title{
Identification of haptoglobin peptide as a novel serum biomarker for lung squamous cell carcinoma by serum proteome and peptidome profiling
}

\author{
TETSUYA OKANO $^{1,2^{*}}$, MASAHIRO SEIKE $^{1 *}$, HIDEHIKO KURIBAYASHI $^{1}$, \\ CHIE SOENO $^{1}$, TAKEO ISHII ${ }^{3}$, KOZUI KIDA ${ }^{3}$ and AKIHIKO GEMMA ${ }^{1}$ \\ ${ }^{1}$ Department of Pulmonary Medicine and Oncology, Graduate School of Medicine, Nippon Medical School, Bunkyo-ku, \\ Tokyo 113-8603; ${ }^{2}$ Department of Thoracic Surgery, Tokyo Medical University, Tokyo 160-0023; \\ ${ }^{3}$ Department of Pulmonary Medicine and Oncology, Graduate School of Medicine, \\ The Respiratory Care Clinic, Nippon Medical School, Tokyo 113-8603, Japan
}

Received August 31, 2015; Accepted November 23, 2015

DOI: 10.3892/ijo.2016.3330

\begin{abstract}
To date, a number of potential biomarkers for lung squamous cell cancer (SCC) have been identified; however, sensitive biomarkers are currently lacking to detect early stage SCC due to low sensitivity and specificity. In the present study, we compared the 7 serum proteomic profiles of 11 SCC patients, 7 chronic obstructive pulmonary disease (COPD) patients and 7 healthy smokers as controls to identify potential serum biomarkers associated with SCC and COPD. Two-dimensional difference gel electrophoresis (2D-DIGE) and mass-spectrometric analysis (MS) using an affinity column revealed two candidate proteins, haptoglobin (HP) and apolipoprotein 4 , as biomarkers of SCC, and $\alpha$-1-antichymotrypsin as a marker of COPD. The iTRAQ technique was also used to identify SCC-specific peptides. HP protein expression was significantly higher in SCC patients than in COPD patients. Furthermore, two HP protein peptides showed significantly higher serum levels in SCC patients than in COPD patients. We established novel polyclonal antibodies for the two HP peptides and subsequently a sandwich enzyme-linked immunosorbent assay (ELISA) for the quantification of these specific peptides in patient and control sera. The sensitivity of detection by ELISA of one HP peptide (HP216) was 70\% of SCC patients, $40 \%$ of COPDs patients and $13 \%$ of healthy controls. We also measured CYFRA, a cytokeratin fragment
\end{abstract}

Correspondence to: Dr Masahiro Seike, Department of Internal Medicine, Division of Pulmonary Medicine/Infection and Oncology, Nippon Medical School, 1-1-5, Sendagi, Bunkyo-ku, Tokyo, 113-8603, Japan

E-mail: mseike@nms.ac.jp

${ }^{*}$ Contributed equally

Key words: lung cancer, serum proteomics, 2D-DIGE, iTRAQ, haptoglobin clinically used as an SCC tumor marker, in all the 28 cases and found CYFRA was detected in only seven SCC cases. However, when the measurement of HP216 was combined with that of CYFRA, 100\% (10 of 10 patients) of SCC cases were detected. Our proteomic profiling demonstrates that the SCC-specific HP peptide HP216 may potentially be used as a diagnostic biomarker for SCC.

\section{Introduction}

Cigarette smoking is a well-known risk factor for the development of certain respiratory diseases, including chronic obstructive pulmonary diseases (COPD), interstitial pulmonary fibrosis and lung cancer (1). Approximately 20-25\% of heavy smokers develop clinically significant COPD while $12-17 \%$ of smokers develop lung cancer. Among these smoking-related pulmonary diseases, lung cancer is the leading cause of cancer death in Japan and also worldwide (2). The odds ratios of lung cancer for Japanese smokers are currently 4.5 for men and 4.2 for females, relative to hospital controls (3). The most common histological types of smoking-related lung cancer are lung squamous cell cancer (SCC) and small cell carcinoma. Of these, the pathogenesis of SCC is the most complex and incompletely understood. In general, targeted molecular agents developed for lung adenocarcinoma are largely ineffective against lung SCC and the current treatment for advanced SCC patients is still cytotoxic chemotherapy. Unfortunately, modern cytotoxic and targeted molecular agents, such as pemetrexed and bevacizumab, are not recommended for patients with SCC $(4,5)$. In addition to smoking being a well-known risk factor for lung cancer, previous findings strongly suggest that COPD is also an important, independent risk factor. For instance, the risk of a lung cancer diagnosis within six months of a COPD diagnosis in patients was reported to be 11-fold greater than in patients without COPD (6). Low-dose helical CT screenings possess the potential to detect early-stage lung cancer and have demonstrated $20 \%$ lower lung cancer mortalities compared to chest X-ray screenings (7). However, it is still difficult to detect lung cancer in a high-risk population using solely radiographic 
examinations. Therefore, the identification of early detection biomarkers for SCC in COPD or heavy smokers is urgently required as this could have a markedly beneficial and clinically significant impact on patient survival.

A number of potential biomarkers for SCC have already been identified; however, sensitive biomarkers are presently unavailable to detect early SCC due to their low sensitivity and specificity. Serum SCC antigen and CYFRA, a cytokeratin fragment, are tumor markers widely used for the evaluation of the therapeutic effects of lung cancer treatments, and in the detection of SCC recurrence, but are not considered applicable to the early detection of SCC $(8,9)$. In addition, no single biomarker, with high enough sensitivity and specificity for the detection of SCC in COPD or heavy smokers, is likely to be discovered.

In the present study, we compared the serum proteomic profiles from SCC cases, COPD patients and healthy smokers to identify any serum biomarkers associated with smokingrelated pulmonary disease, including SCC and COPD. Serum is a highly complex bodily fluid that contains proteins ranging in concentrations over at nine orders of magnitude (10). Since high abundance proteins can interfere in the separation and detection of low abundance proteins, an immunoaffinity column was used to deplete highly abundant plasma proteins, and the resulting $>2000$ protein spots were separated by twodimensional difference gel electrophoresis (2D-DIGE) and mass-spectrometry analysis (MS) (11). After isobaric tagging for relative and absolute quantification (iTRAQ)-2DLC-MS/ MS experiments (12), we identified candidate proteins as serum biomarkers of SCC by 2D-DIGE and western blot analysis. Using enzyme-linked immunosorbent assay (ELISA), we identified a cancer-associated serum peptide of haptogloblin (HP), haptoglobin 216 (HP 216), as a novel biomarker for SCC.

\section{Materials and methods}

Serum samples. The present study was approved by the institutional review boards of the Nippon Medical School Hospital and Nippon Medical School Respiratory Care Clinic. All patients provided informed consent. Serum samples for 2D-DIGE and MS analysis were obtained from 11 SCC patients at Nippon Medical School Hospital, and 7 COPD patients and 7 healthy smokers at Nippon Medical School Respiratory Care Clinic, all of whom were $<60$ years of age. The 25 cases were all male patients or healthy volunteers, with a smoking history of 15 or more pack-years. The first set of serum samples collected consisted of 12 samples and the second set consisted of 13 samples. We also used an additional 23 serum samples, for ELISA, collected from five SCC patients at Nippon Medical School Hospital, 10 COPD patients and eight healthy smokers at Nippon Medical School Respiratory Care Clinic, all of whom were $<70$ years of age. After blood sampling, each serum was immediately separated by centrifugation at $1,500 \mathrm{rpm}$ for $15 \mathrm{~min}$ at room temperature. Aliquots of sera were taken and stored at $-80^{\circ} \mathrm{C}$ until use.

Removal of high-abundance proteins from serum samples and 2D-DIGE. Serum samples were processed using an affinity column (Agilent spin concentrators; Agilent Technologies, Santa Clara, CA, USA), which was expected to selectively remove abundant proteins, including albumin, IgG, IgA, transferrin and HP, from serum samples, according to the manufacturer's instructions. Protein samples were then labeled with cysteine reactive saturation dye (GE Healthcare, Buckinghamshire, UK). The labeling reaction was performed according to the manufacturer's instructions as previously described (13). An internal serum control mixture was made by mixing equal portions of three or four healthy control samples. The internal control sample was labeled with $\mathrm{Cy} 3$ and patient samples were labeled with Cy5 and 2D-DIGE was performed as previously described (13-16). Each labeled protein sample was loaded onto triplicate IPG DryStrip gels $(24 \mathrm{~cm}$ length; pI values ranging from 4 to 7 ; GE Healthcare). The equilibrated IPG gels were applied onto $12 \%$ polyacrylamide gels (GE Healthcare), and proteins were then separated using an Ettan DALT-six electrophoresis system (GE Healthcare). All gel images were scanned on a Typhoon 9400 Imager (GE Healthcare). Statistics and the quantitation of protein expression were carried out using DeCyder 2-D differential analysis software (GE Healthcare). A paired t-test derived P-value of $<0.05$ was considered statistically significant.

Mass spectrometry. To identify proteins corresponding to spots on 2D gels, mass spectrometry was performed. An automated spot recovery robot, the Ettan Spot Picker (GE Healthcare), transferred protein spots from each gel to a 96-well plate. In-gel digestion was then performed as previously described and tryptic peptides were subjected to mass spectrometric analysis (13-16). Peptide map fingerprinting (PMF) analysis was performed with ESI-LC/Q-TOF-MS (Waters-Micromass) (LabX Media Group Inc., Midland, ON, Canada). Mass spectra were processed using Analyst QS software and a Mascot program. We performed a second Swiss-Prot database search.

Western blot analysis. Protein $(10 \mu \mathrm{g})$ was separated by gel electrophoresis on $10 \%$ gels, transferred to nitrocellulose membranes and detected by immunoblotting using specific antibodies and a chemiluminescence system (GE Healthcare). Antibodies detecting HP, apolipoprotein (APOA4), $\alpha$-1-antichymotrypsin, (AACT) and fibronection were purchased from Abcam (Cambridge, UK).

$i T R A Q-2 D L C-M S / M S$. Protein identification and quantification for iTRAQ-2DLC-MS/MS experiments was carried out using ProteinPilot 2.0 software according to the manufacturer's protocols (Filgen, Inc., Nagoya, Japan) (12). Proteins were labeled with iTRAQ tags as follows: SCC samples with a 116 isobaric tag; and healthy smoker samples with a 114 isobaric tag. A protein sequence search was performed using a Swiss-Prot human database. At least two peptides with $95 \%$ confidence were considered for protein identification and results were then exported into Excel for manual data interpretation. Protein quantitation for iTRAQ were carried out using ProteinPilot 2.0 software. One-sample t-test derived P-value of $<0.05$ was considered statistically significant and shown in Table II.

Establishment of polyclonal antibodies for HP peptides and a HP ELISA. Customized, rabbit anti-HP peptide antibodies against two peptides were raised (PharmaBio, Nagoya, Japan). 
Table I. The differentially expressed proteins in SCC and COPD.

\begin{tabular}{|c|c|c|c|c|c|c|}
\hline \multirow[b]{2}{*}{ Spot no. } & \multirow[b]{2}{*}{ Protein ID } & \multirow[b]{2}{*}{ Protein name } & \multicolumn{2}{|c|}{1 st set } & \multicolumn{2}{|c|}{ 2nd set } \\
\hline & & & Ratio & P-value & Ratio & P-value \\
\hline \multicolumn{7}{|c|}{ SCC vs. COPD } \\
\hline 1289 & gil306882 & Haptoglobin precursor & 10.4 & $2.6 \mathrm{E}-06$ & 4.2 & 0.008 \\
\hline 1325 & gil306882 & Haptoglobin precursor & 4.9 & $9.1 \mathrm{E}-06$ & 8.1 & 0.009 \\
\hline 1341 & gil306882 & Haptoglobin precursor & 4.0 & 0.000053 & 8.7 & 0.003 \\
\hline 1307 & gil178759 & Apolipoprotein A-IV & 10.4 & $1.8 \mathrm{E}-06$ & 6.7 & 0.005 \\
\hline 1313 & gil71773110 & Apolipoprotein A-IV precursor & 5.9 & 0.000042 & 9.6 & 0.005 \\
\hline 1991 & gil78101268 & Complement component $\mathrm{C} 3$ & 5.2 & 0.000032 & 2.8 & 0.006 \\
\hline 1897 & gil337758 & Pre-serum amyloid P component & 8.7 & 0.000018 & 3.4 & 0.007 \\
\hline 1920 & gil28317 & Unnamed protein product & 5.7 & 0.000011 & 1.7 & 0.04 \\
\hline 1925 & gil7959223 & KIAA1481 protein & 12.0 & $2.1 \mathrm{E}-07$ & 3.8 & 0.02 \\
\hline 1934 & gil11935049 & Keratin 1 & 9.2 & $1.4 \mathrm{E}-06$ & 2.8 & 0.006 \\
\hline \multicolumn{7}{|c|}{ COPD vs. smoker } \\
\hline 1963 & gil7331218 & Keratin 1 & 4.7 & 7.5E-008 & 1.2 & 0.04 \\
\hline 804 & gil177809 & $\alpha-1$-antichymotrypsin & -5.7 & $8.2 \mathrm{E}-006$ & -1.7 & 0.04 \\
\hline 846 & gil177809 & $\alpha-1$-antichymotrypsin & -4.7 & $1.2 \mathrm{E}-006$ & -2.7 & 0.001 \\
\hline 792 & gil177809 & $\alpha-1$-antichymotrypsin & -3.9 & $1.3 \mathrm{E}-006$ & -1.8 & 0.04 \\
\hline
\end{tabular}

Serum samples were eluted from the affinity column, which was expected to selectively remove HP from serum samples (Agilent spin concentrators; Agilent Technologies). Duplicates of 28 sera samples from SCC and COPD patients, and smokers, as well as positive and negative haptoglobin peptide controls, were coated overnight onto a 96 -well plate at $4^{\circ} \mathrm{C}$. Each well was washed twice in PBS and blocked with 3\% BSA/PBS for $1 \mathrm{~h}$ at $37^{\circ} \mathrm{C}$. After washing twice, rabbit anti-HP peptide antibodies (1:1,000 dilution) were added to wells and incubated for $2 \mathrm{~h}$ at $37^{\circ} \mathrm{C}$. Subsequently, FITC-labeled goat anti-rabbit $\mathrm{IgG}$ antibody was added to the wells for $1 \mathrm{~h}$ at $37^{\circ} \mathrm{C}$. Finally, the plate was read at a wavelength of $535 \mathrm{~nm}$ on a fluorescence plate reader (Perkin-Elmer, Inc., Waltham, MA, USA).

Immunoradiometric assay for CYFRA. Plasma (80 $\mu \mathrm{l})$ was analyzed using a commercially available TM-CYFRA 21-1 ELISA kit (DRG Instruments GmbH, Marburg, Germany) according to the manufacturer's recommendations.

Statistical analysis. The set of spot intensities and prior biological information were used in statistical analyses. Data from 2D-DIGE was analyzed by principal component analysis (PCA) using Impressionist software (Genedata AG, Basel, Switzerland) $(13,14)$. PCA is a multidimensionality-reduction technique used to visualize similarities and differences between samples. It belongs to the class of unsupervised methods.

\section{Results}

Identification of proteins in smoking-related pulmonary disease by $2 D$-DIGE. We compared serum protein expression profiles of SCC and COPD patients with those of healthy smokers. Serum samples were collected from young adult men ( $<60$ years) with a moderate to heavy smoking habit $(>15$ pack years) but without comorbidity. An immunoaffinity column was used to remove abundant serum proteins and quantitative protein profiles of samples were subsequently generated by 2D-DIGE. More than 2400 spots were resolved, and computerassisted quantitative analysis was performed.

Fig. 1A illustrates a representative Cy5 image unique to SCC patients. To estimate the ability of 2D-DIGE profiling to distinguish between patients based on the types of smokingrelated diseases, PCA was performed using a data set of protein spot profiles. Twelve patients of the first set of serum samples collected were divided into three groups according to patients types (Fig. 1B). Thirteen patients of the second collected set of serum samples were also divided into three groups according to patient types (Fig. 1B). We tried to identify differentially expressed protein spots common to both cohorts. First, we identified 30 serum protein spots that distinguished SCC from COPD patients using the first set. We validated these protein expression levels using an independent, second sample set. Of the 30 spots, we identified 10 SCC serum-specific protein spots with increased intensity when compared with COPD serum in the second set (Fig. 1A and C). To more specifically identify those 10 differentially expressed proteins common to both cohorts, peptide mass fingerprinting of tryptic digests was performed by mass spectrometry. A database search successfully identified 10 proteins with expression significantly higher in SCC than in COPD sera (Table I and Fig. $1 \mathrm{C} ; \mathrm{P}<0.05$ for all). Three spots were identified as HP protein and a further two were identified as apolipoprotein A-IV (APOA4). We also identified 4 COPD serum-specific protein spots when compared with samples from smokers in both sets of collected sera (Table I and Fig. 1A). Three of these spots were $\alpha$-1-antichymotrypsin 


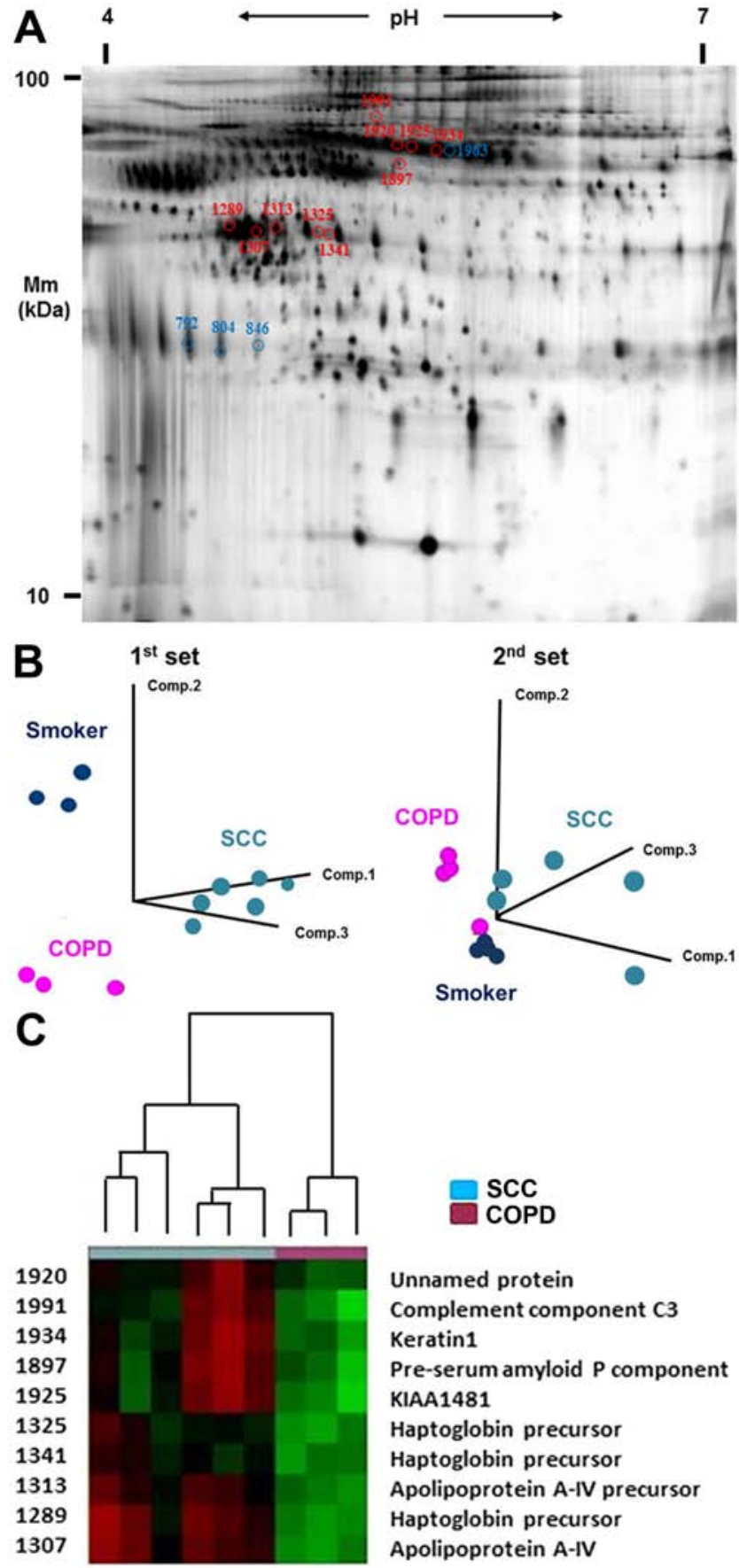

(AACT), whose expression was significantly lower in COPD than in the sera of smokers $(\mathrm{P}<0.05$ for all).

To confirm the degree of change in spot intensity observed by 2D-DIGE, protein samples of the first and second sets, after affinity column treatment to remove abundant serum proteins, including HP, were separated by SDS-PAGE, western blotted and incubated with antibodies against HP. The detection of HP protein bands had not been expected at the start of this study because of the expected removal of all high-abundance proteins, including HP, by the affinity column and this was confirmed in COPD and healthy smoker sera by western blotting. However, the expression of HP was detected in all five SCC patient serum samples, in spite of the removal of highabundance proteins (Fig. 1D). In addition, high molecular weight isoforms of APOA4, probably due to post-translational
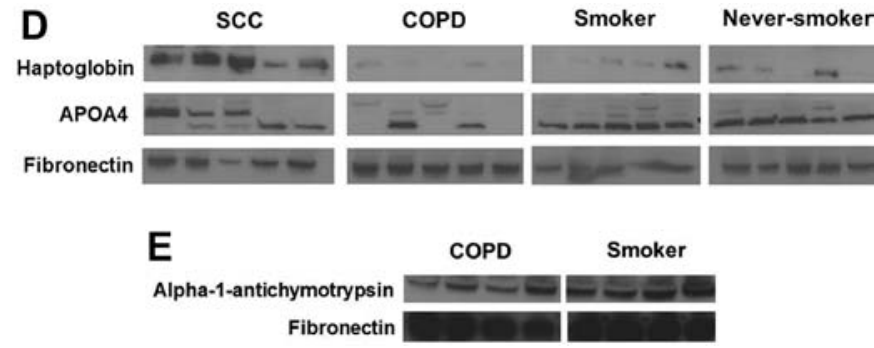

Figure 1. Statistical analysis of 25 serum samples as a function of their protein expression. Profiles (A) A representative 2-D image of a Cy5-labeled proteins of SCC patients. Spots with increased intensity on SCC patients are marked by red circles. Spots with increased intensity on COPD patients are marked by blue circles. Spot numbers correspond to those in Table I. (B) A three-dimensional plot of the results of principal component analysis (PCA) using 2520 unselected spots in the first and second sets of collected serum samples. (C) Hierarchical clustering analysis of the 10 selected protein spots showing a significant difference between SCC and COPD cases in both sets. SCC and COPD cases are clearly divided into groups according to their organ of origin. Protein names are listed on the right. (D and E) Western blot analyses for the validation of alterations. Serum protein samples were separated by SDS-PAGE and western blots incubated with antibodies against haptoglobin, apolipoprotein IV (APO4), $\alpha$-1-anti-chymotrypsin, and fibronectin. Fibronectin was used as a loading control.

modifications, were also found in three of five SCC samples (Fig. 1D). In COPD cases, the decreased expression of $\alpha$-1-antichymotrypsin (AACT) was observed by western blotting (Fig. 1E). These results suggest that HP and APO-A4 may be involved in the carcinogenesis of SCC, and the decreased expression of AACT may contribute to the occurrence of COPD.

Identification, by iTRAQ-2DLC-MS/MS, of peptides whose expression is affected by the smoking-related pulmonary disease. To further identify specific peptides or proteins with post-translational modifications to distinguish SCC patients from COPD patients, iTRAQ-2DLC-MS/MS was performed. Using pooled serum samples from SCC and COPD patients. A total of 861 proteins were filtered and quantified using manually selected filter exclusion parameters. An additional 2.0 -fold change cut-off for all iTRAQ ratios (ratio $>2.0$ or $<0.5$ ) was selected to classify proteins as upregulated or downregulated in SCC. Sixteen proteins were screened from SCC samples, including 12 and 4 proteins upregulated and downregulated in SCC, respectively $(\mathrm{P}<0.05$ for all; error factor $[\mathrm{EF}]<2$; Table II). Notably, HP protein expression was significantly increased by $>10.8$-fold in the SCC sample $(\mathrm{P}<0.001)$. A total of 187 peptides were used for the identification of HP protein $(E F=1.25)$. Among these identified HP peptides, two peptides were located in the $\mathrm{N}$-terminal region of the $\mathrm{Hp} \beta$-chain, namely, HP60-72 (HP60) and HP216-228 (HP216) (Fig. 2A), and showed significantly higher serum levels in SCC patients than in COPD patients (Fig. 2B and C). A 11.3-fold increase in levels of the HP60 peptide sequence was observed in SCC compared to COPD samples (Fig. 2B). The HP216 peptide sequence was also increased by 21.3 -fold in SCC compared to COPD samples (Fig. 2C). As for APOA4, protein expression levels between SCC and COPD samples remained unchanged according to the iTRAQ method (results not shown). In addition, specific post-translational modifications, including 
A 1 MSALGAVIALLLWGQLFAVDSGNDVTDIADDGCPKPPEIAHGYVEHSVRYQCKNYYKLRT

61 EGDGVYTLNDKKQWINKAVGDKLPECEADDGCPKPPEIAHGYVEHSVRYQCKNYYKLRTE

121 GDGVYTLNNEKQWINKAVGDKLPECEAVCGKPKNPANPVQRILGGHLDAKGSFPWQAKMV

181 SHHNLTTGATLINEQWLLTTAKNLFLNHSENATAKDIAPTLTLYVGKKQLVEIEKVVLHP

241 NYSQVDIGLIKLKQKVSVNERVMPICLPSKDYAEVGRVGYVSGWGRNANFKFTDHLKYVM

301 LPVADQDQCIRHYEGSTVPEKKTPKSPVGVQPILNEHTFCAGMSKYQEDTCYGDAGSAFA

361 VHDLEEDTWYATGILSFDKSCAVAEYGVYVKVTSIQDWVQKTIAEN

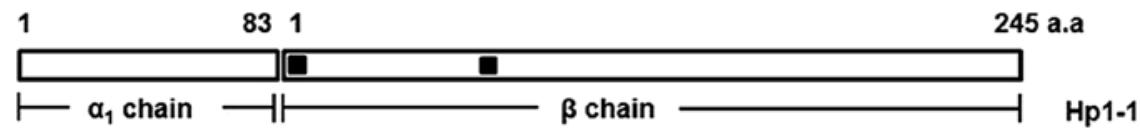

\section{B TEGDGVYTLNDKK SCC: $C O P D=11,3: 1$}
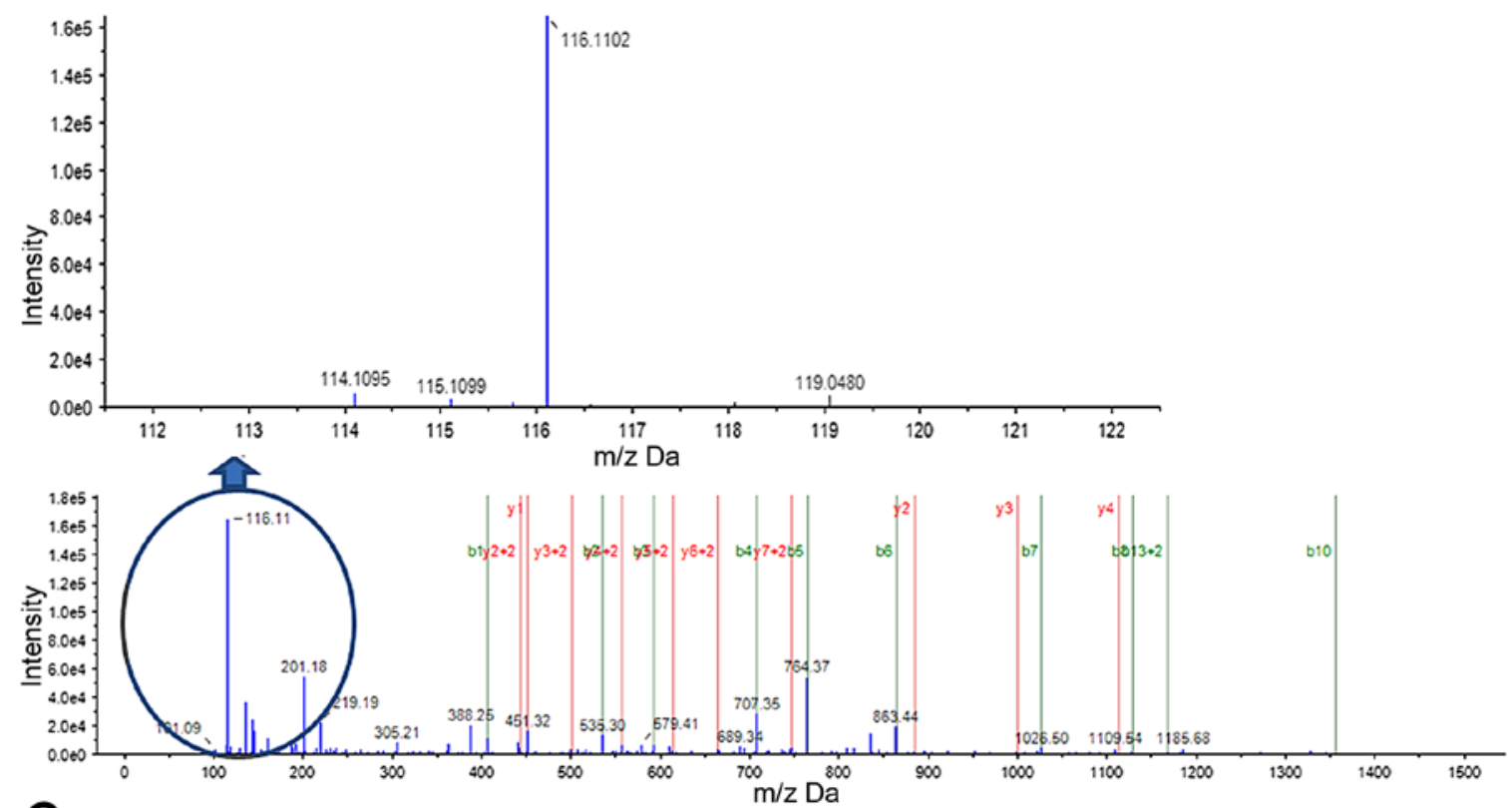

C DIAPTLTLYVGKK SCC:COPD $=21.3: 1$
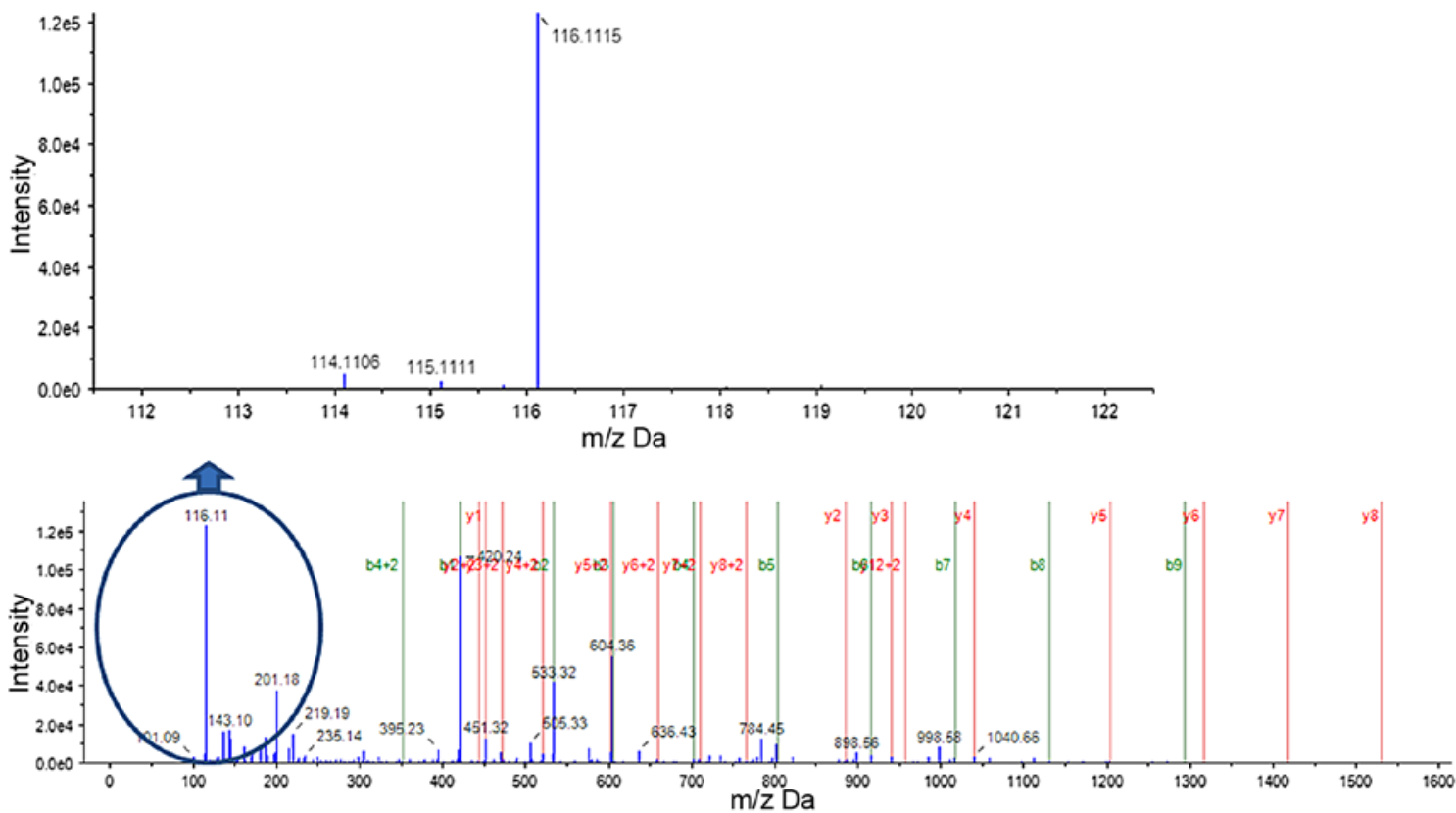

Figure 2. HP protein sequence. (A) Aligned HP 406 amino acid sequences. Two significantly overexpressed peptides were detected by iTRAQ and sequences are shown in red font. The two peptides are located in the N-terminal region of the $\beta$-chain of the HP phenotype (HP 1-1). (B and C) Representative MS/MS spectra showing the peptides of the HP protein. The MS/MS spectra show the identified peptide sequences, TEGDGVYTLNDKK or DIAPTLTLYVGKK, leading to the identification of HP. 
Table II. Protein expression levels, after iTRAQ analysis, in SCC and COPD cases.

\begin{tabular}{|c|c|c|c|c|c|}
\hline Accession no. & Protein name & Peptides $(95 \%)$ & $116: 114$ & P-value & $\mathrm{EF}$ \\
\hline IPI00641737.2 & Haptoglobin & 187 & 10.8 & $<0.001$ & 1.25 \\
\hline IPI00553177.1 & Isoform 1 of $\alpha$-1-antitrypsin & 15 & 5.4 & $<0.001$ & 1.45 \\
\hline IPI00022429.3 & $\alpha$-1-acid glycoprotein 1 & 164 & 3.2 & $<0.001$ & 1.56 \\
\hline IPI00027462.1 & Protein S100-A9 & 6 & 3.1 & 0.002 & 1.50 \\
\hline IPI00022417.4 & Leucine-rich $\alpha$-2-glycoprotein & 56 & 2.9 & $<0.001$ & 1.20 \\
\hline IPI00876950.1 & Isoform 2 of inter- $\alpha$-trypsin inhibitor heavy chain $\mathrm{H} 3$ & 51 & 2.9 & $<0.001$ & 1.18 \\
\hline IPI00478003.3 & $\alpha$-2-macroglobulin & 825 & 2.7 & $<0.001$ & 1.14 \\
\hline IPI01009486.1 & Uncharacterized protein & 11 & 2.5 & $<0.001$ & 1.33 \\
\hline IPI00022395.1 & Complement component C9 & 34 & 2.4 & $<0.001$ & 1.32 \\
\hline IPI00896380.1 & Isoform 2 of $\mathrm{Ig}$ mu chain $\mathrm{C}$ region & 14 & 2.3 & $<0.001$ & 1.15 \\
\hline IPI01025667.1 & $\alpha$-1-antitrypsin & 141 & 2.3 & $<0.001$ & 1.24 \\
\hline IPI00296608.6 & Complement component C7 & 34 & 2.0 & $<0.001$ & 1.32 \\
\hline IPI00064667.5 & $\beta$-Ala-His dipeptidase & 20 & 0.5 & $<0.001$ & 1.16 \\
\hline IPI00339228.2 & Fibronectin & 126 & 0.4 & $<0.001$ & 1.23 \\
\hline IPI00657670.1 & Apolipoprotein C-III variant 1 & 40 & 0.4 & 0.006 & 1.59 \\
\hline IPI00216065.3 & Isoform 2 of Vitamin $\mathrm{K}$-dependent protein $\mathrm{Z}$ & 2 & 0.4 & 0.004 & 1.45 \\
\hline
\end{tabular}
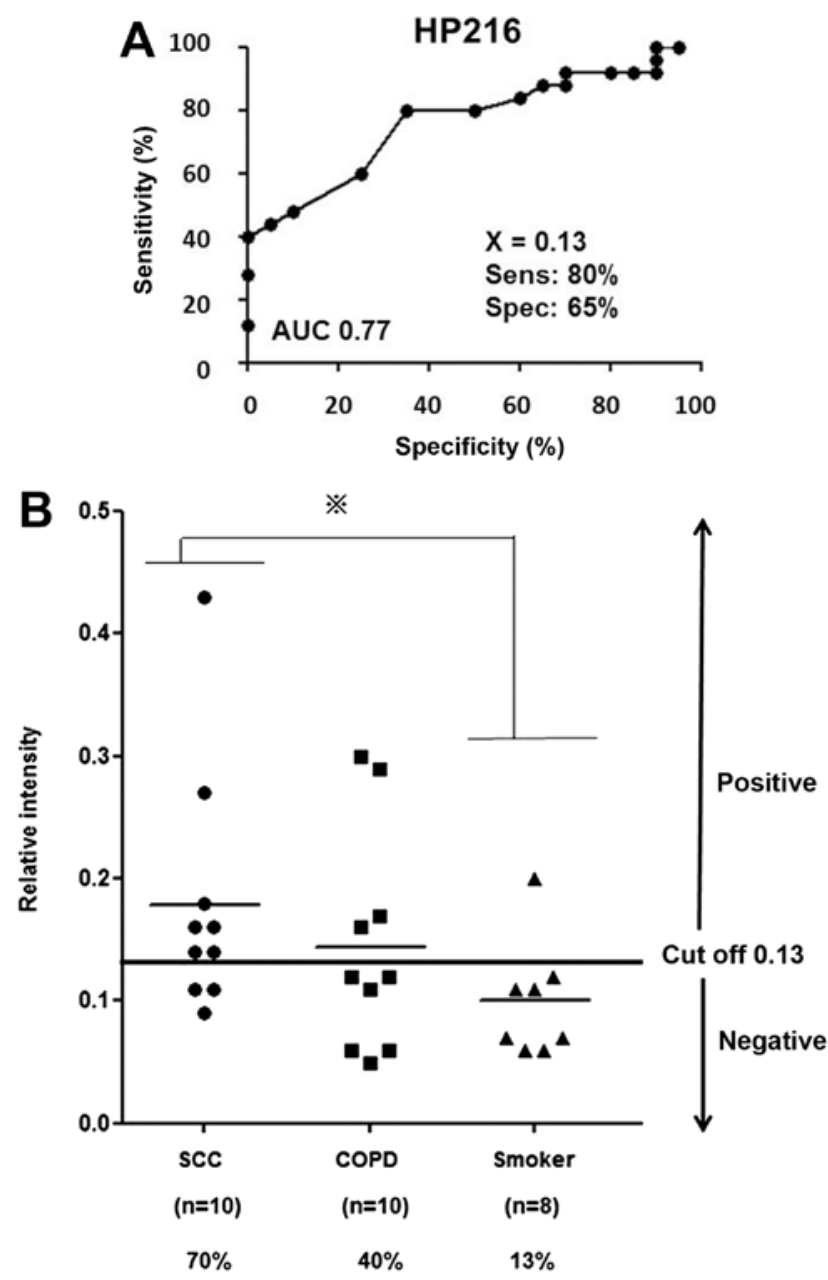

Figure 3. HP peptide ELISA. (A) A ROC curve and AUC values showing the discriminating capacities of HP216. (B) HP216 peptide expression by ELISA. HP216 peptide levels were significantly higher in sera from SCC patients than in healthy smokers. A Mann-Whitney test showed a significant statistical difference between the SCC $(n=10)$ compared to the healthy controls group $(\mathrm{n}=8) .{ }^{*} \mathrm{P}<0.05$
Table III. Detection rates for CYFRA and HP216, and their combination.

\begin{tabular}{lcccc}
\hline & $\begin{array}{c}\text { No. of } \\
\text { cases }\end{array}$ & $\begin{array}{c}\text { CYFRA } \\
\mathrm{n},(\%)\end{array}$ & $\begin{array}{c}\text { HP216 } \\
\mathrm{n},(\%)\end{array}$ & $\begin{array}{c}\text { Combination } \\
\mathrm{n},(\%)\end{array}$ \\
\hline SCC & 10 & $7(70)$ & $7(70)$ & $10(100)$ \\
COPD & 10 & $0(0)$ & $4(40)$ & \\
Smoker & 8 & $0(0)$ & $1(13)$ & \\
\hline
\end{tabular}

phosphorylation of APOA4, were not found. These results suggest that two specific HP peptide sequences were candidate serum biomarkers for SCC.

HP peptide ELISA. We next assessed the sensitivity and specificity of these two peptides for SCC diagnosis by receiver operating characteristic (ROC) curve analysis. The cut-off value was set at the point whose distance from the (sensitivity, specificity) $=(1,1)$ reached the minimum (Fig. 3A). To assess the quantitative reproducibility of these two peptides, we conducted further validation studies by ELISA using 28 serum samples consisting of five available SCC samples from the second set of 2D-DIGE and 23 additional serum samples. We first established novel polyclonal antibodies for HP60 and HP216 and then developed a sandwich ELISA for the quantification of these specific peptides using samples from 10 SCC and 10 COPD patients, and eight healthy control smokers. For HP60, expression levels of the peptide were identical between SCC and healthy control serum samples (data not shown). In contrast, significantly high levels of HP216 peptide were found in sera from SCC patients when compared with those of healthy smoker controls (Fig. 3B; $\mathrm{P}<0.05$ ). The sensitivity of HP216 (cut-off value of 0.13 ) was $70 \%$ (7 of 10) of SCC patients, $40 \%$ (4 of 10) of COPD patients and 13\% (1 of 8) of 
Table IV. The results of CYFRA and HP 216, and their combination.

\begin{tabular}{lrrrrc}
\hline No. & Case & CYFRA & HP216 & Combination \\
\hline 1 & SCC & $14.8+$ & $0.14+$ & + \\
2 & SCC & $14.4+$ & $0.16+$ & + \\
3 & SCC & $10.9+$ & $0.14+$ & + \\
4 & SCC & $9.1+$ & $0.18+$ & + \\
5 & SCC & $70.1+$ & $0.11-$ & + \\
6 & SCC & $39.6+$ & $0.09-$ & + \\
7 & SCC & $6.1+$ & $0.11-$ & + \\
8 & SCC & $2.3-$ & $0.43+$ & + \\
9 & SCC & $2.0-$ & $0.16+$ & + \\
10 & SCC & $1.8-$ & $0.27+$ & + \\
\hline
\end{tabular}

smoker controls (Fig. 3B). These results suggest that HP216 is a novel candidate serum biomarker of SCC patients.

Immunoradiometric assay for CYFRA. We also measured serum CYFRA protein levels, clinically used as SCC tumor marker, in all 28 cases. The sensitivity of CYFRA protein level (cut-off value of 3.0) in serum samples of SCC, and COPD patients, and healthy controls was $70 \%$ (7 of 10), $0 \%$ (0 of 10 ) and $0 \%$ (0 of 8), respectively (Table III). Interestingly, the detection of HP216 peptides was positive in three CYFRA false-negative SCC cases (Table IV). When the detection of HP216 was combined with that of CYFRA, 100\% (10 of 10 patients) of SCC cases were detected (Table IV). These findings suggest that the SCC-specific peptide HP216 may be used as a diagnostic biomarker for SCC. A combination of detecting HP216 with CYFRA protein fragment levels in patient sera may be applicable to diagnose SCC.

\section{Discussion}

Smoking history and the existence of COPD are important risk factors for SCC $(1,3)$. However, it is not easy to detect SCC in COPD patients and heavy smokers by the existing method of radiographic examination. Targeted molecular therapies, such as epidermal growth factor receptor (EGFR) tyrosinekinase inhibitor (EGFR-TKI) and anaplastic lymphoma kinase (ALK) inhibitor, for lung adenocarcinoma have been developed (17-19); however, SCC shows resistance to standard chemotherapy and targeted molecular therapy $(4,5)$. Therefore, serum biomarkers have great potential to facilitate the early detection of SCC in patients with SCC risk factors such as COPD and a smoking history, and for the monitoring of cancer recurrence and treatments in SCC patients, resulting in improvements in the prognosis for this disease.

Chronic tobacco smoking is a major risk factor for the development of COPD, but only a relatively small proportion of smokers actually develop airway obstruction. It is well known that $\alpha-1$ antitrypsin deficiency (AATD) is a hereditary disorder characterized by low circulating levels of the key antiprotease, $\alpha-1$ antitrypsin, and is associated with the development of COPD, often by the third or fourth decade (20). In this study, we found that decreased serum expression of AACT was observed in COPD patients. AACT belongs to the class of serine protease inhibitors and is synthesized in the liver by alveolar macrophages and airway epithelia (21). Several cases of COPD were reported to be associated with mutations and polymorphisms in the AACT gene $(22,23)$. Although the frequency of AATD is relatively low in Japanese patients, as is the case with $\alpha-1$ antitrypsin, decreased serum expression of AACT in heavy smokers may contribute to the development of COPD in Japanese.

In the present study, we compared the serum protein profiles, using 2D-DIGE/MS and iTRAQ/MS analyses, of SCC, COPD, and healthy smoker cases of identical ages to identify a biomarker for SCC and COPD patients. In general, a relatively small number of $\sim 200-300$ protein spots obtained by 2D-DIGE from serum samples have, for a long time, been a major concern (10); employing an affinity column was found to significantly improve the number of protein spots detected to over 2000 spots. The iTRAQ-based approach covered peptide profiling, which could not be done by 2D-DIGE (12). Based on two independent proteomics techniques followed by ELISA, total HP or HP peptide (HP 216), was identified as a candidate serum biomarker of SCC. Furthermore, SCC was predicted in all ten SCC patients only when using the detection of HP216 combined with CYFRA.

We specifically focused on HP as a biomarker of SCC. HP is classified as an acute phase protein whose serum expression levels increases with inflammation, infection, and several cancers in this study (24). The major site for HP biosynthesis is the liver and its synthesis is regulated by various inflammatory cytokines, including IL-1, IL- 6 , TNF- $\alpha$ and TNF- $\beta$. Previous studies have demonstrated increases in the serum level of total HP protein and HP fragments, as well as specific HP post-translational modifications, including fucosylation and glycosylation, in lung and colorectal cancer patients (25-28). Abnormal HP glycosylation was found to interfere with tumor metastasis (27), while fucosylated HP was shown to be a novel biomarker for colorectal cancer (28). In addition, HP has been shown to be an angiogenic factor in sera from patients with vasculitis and cancer. It is likely that the causes of these changes may encompass host-defense and tumor-promoting mechanisms. By processing serum samples after the removal of high-abundance proteins, we finally identified HP216 peptide as a candidate SCC biomarker. Both the origin and function of HP216 are still unknown; however, serum levels of a two-residue longer peptide, HP216-230, were also significantly higher in SCC the patient samples in the present study (data not shown). CYFRA21-1 is a fragment of cytokeratin 19 that has been extensively studied in patients with SCC and has been demonstrated to be clinically useful (9). These findings suggest the existence of SCC-associated endo- or exopeptidases responsible for cleavage at HP amino acid 216 in SCC patients.

No single biomarker will be able to detect all SCC cases with a high enough specificity and sensitivity. Recent study demonstrated that combination detection of HP and current tumor markers could significantly improve the sensitivity and specificity in diagnosis of lung cancer (29). Four tumor markers including CEA, NSE, CYFRA as well as HP the combined together could produce a positive detection rate of 
$85 \%$, significantly higher than that of any single test (29). In the present study, all SCC cases were detected by the measurement of HP216 combined with CYFRA. A combination of detecting serum HP216 with CYFRA levels may be applicable to diagnose SCC.

Taken together, the HP peptide HP216, is a promising cancer biomarker for the early detection of SCC in highrisk lung cancer populations, including COPD patients and heavy smokers. Combined with CYFRA determinations, the measurement of serum HP216 may lead to the earlier detection of SCC in at-risk patients and become a novel diagnostic tool for SCC. A limitation of the study was the relatively small sample size. Further studies will be needed to confirm the significance of HP216 using a large number of subjects and to elucidate the origin and function of the HP peptide.

\section{Acknowledgements}

We thank Dr Tadashi Kondo at Division of Rare Cancer Research, National Cancer Center Research Institute for technical advice and helpful discussion. We also thank Ms. Mina Fujishiro for sample collection. The present study was supported in part by a Grant-in-Aid from Smorking Research Fundation (to A.G.).

\section{References}

1. JCS Joint Working Group; Japanese Society for Oral Health; Japanese Society of Oral and Maxillofacial Surgeons; Japanese Society of Public Health; Japanese Respiratory Society; Japan Society of Obstetrics and Gynecology; Japanese Circulation Society; Japan Pediatric Society; Japanese College of Cardiology; Japan Lung Cancer Society: Guidelines for Smoking Cessation (JCS 2010) - digest version. Circ J 76: 1024-1043, 2012.

2. Siegel R, Naishadham D and Jemal A: Cancer statistics 2013. CA Cancer J Clin 63: 11-30, 2013

3. Sobue T, Yamamoto S, Hara M, Sasazuki S, Sasaki S and Tsugane S; JPHC Study Group; Japanese Public Health Center: Cigarette smoking and subsequent risk of lung cancer by histologic type in middle-aged Japanese men and women: the JPH study. Int J Cancer 99: 245-251, 2002.

4. Scagliotti GV, Parikh P, von Pawel J, Biesma B, Vansteenkiste J, Manegold C, Serwatowski P, Gatzemeier U, Digumarti R, Zukin $\mathrm{M}$, et al: Phase III study comparing cisplatin plus gemcitabine with cisplatin plus pemetrexed in chemotherapynaive patients with advanced-stage non-small-cell lung cancer. J Clin Oncol 26: 3543-3551, 2008.

5. Sandler A, Gray R, Perry MC, Brahmer J, Schiller JH, Dowlati A, Lilenbaum R and Johnson DH: Paclitaxel-carboplatin alone or with bevacizumab for non-small-cell lung cancer. N Engl J Med 355: 2542-2550, 2006.

6. Powell HA, Iyen-Omofoman B, Baldwin DR, Hubbard RB and Tata LJ: Chronic obstructive pulmonary disease and risk of lung cancer: The importance of smoking and timing of diagnosis. $\mathrm{J}$ Thorac Oncol 8: 6-11, 2013.

7. Kovalchik SA, Tammemagi M, Berg CD, Caporaso NE, Riley TL, Korch M, Silvestri GA, Chaturvedi AK and Katki HA: Targeting of low-dose CT screening according to the risk of lung-cancer death. N Engl J Med 369: 245-254, 2013.

8. Torre GC: SCC antigen in malignant and nonmalignant squamous lesions. Tumour Biol 19: 517-526, 1998.

9. Pujol JL, Grenier J, Daurès JP, Daver A, Pujol H and Michel FB: Serum fragment of cytokeratin subunit 19 measured by CYFRA 21-1 immunoradiometric assay as a marker of lung cancer. Cancer Res 53: 61-66, 1993.

10. Tirumalai RS, Chan KC, Prieto DA, Issaq HJ, Conrads TP and Veenstra TD: Characterization of the low molecular weight human serum proteome. Mol Cell Proteomics 2: 1096-1103, 2003.
11. Okano T, Kondo T, Kakisaka T, Fujii K, Yamada M, Kato H, Nishimura T, Gemma A, Kudoh S and Hirohashi S: Plasma proteomics of lung cancer by a linkage of multi-dimensional liquid chromatography and two-dimensional difference gel electrophoresis. Proteomics 6: 3938-3948, 2006.

12. Latterich M, Abramovitz M and Leyland-Jones B: Proteomics: New technologies and clinical applications. Eur J Cancer 44: 2737-2741, 2008 .

13. Seike M, Kondo T, Fujii K, Okano T, Yamada T, Matsuno Y, Gemma A, Kudoh S and Hirohashi S: Proteomic signatures for histological types of lung cancer. Proteomics 5: 2939-2948, 2005.

14. Seike M, Kondo T, Fujii K, Yamada T, Gemma A, Kudoh S and Hirohashi S: Proteomic signature of human cancer cells. Proteomics 4: 2776-2788, 2004.

15. Seike M, Kondo T, Mori Y, Gemma A, Kudoh S, Sakamoto M, Yamada $\mathrm{T}$ and Hirohashi S: Proteomic analysis of intestinal epithelial cells expressing stabilized beta-catenin. Cancer Res 63: 4641-4647, 2003.

16. Okano T, Kondo T, Fujii K, Nishimura T, Takano T, Ohe Y, Tsuta K, Matsuno Y, Gemma A, Kato H, et al: Proteomic signature corresponding to the response to gefitinib (Iressa, ZD1839), an epidermal growth factor receptor tyrosine kinase inhibitor in lung adenocarcinoma. Clin Cancer Res 13: 799-805, 2007.

17. Maemondo M, Inoue A, Kobayashi K, Sugawara S, Oizumi S, Isobe $\mathrm{H}$, Gemma A, Harada M, Yoshizawa H, Kinoshita I, et al: Gefitinib or chemotherapy for non-small-cell lung cancer with mutated EGFR. N Engl J Med 362: 2380-2388, 2010.

18. Mitsudomi T, Morita S, Yatabe Y, Negoro S, Okamoto I, Tsurutani J, Seto T, Satouchi M, Tada H, Hirashima T, et al; West Japan Oncology Group: Gefitinib versus cisplatin plus docetaxel in patients with non-small-cell lung cancer harbouring mutations of the epidermal growth factor receptor (WJTOG3405): An open label, randomised phase 3 trial. Lancet Oncol 11: 121-128, 2010.

19. Solomon BJ, Mok T, Kim DW, Wu YL, Nakagawa K, Mekhail T, Felip E, Cappuzzo F, Paolini J, Usari T, et al: First-line crizotinib versus chemotherapy in ALK-positive lung cancer. N Engl J Med 371: 2167-2177, 2014.

20. Kelly E, Greene CM, Carroll TP, McElvaney NG and O'Neill SJ: Alpha-1 antitrypsin deficiency. Respir Med 104: 763-772, 2010.

21. Nagareda T, Takeda M, Kojima K, Tanaka A, Terada N, Yamasaki T, Nagareda T, Ueno $\mathrm{H}$ and Kotoh K: Alpha-1 antichymotrypsin is increased in human alveolar macrophages by phorbol myristate acetate or lipopolysaccharide and released from these activated macrophages by glucocorticoid. J Pathol 165: 319-323, 1991.

22. Poller W, Faber JP, Weidinger S, Tief K, Scholz S, Fischer M, Olek K, Kirchgesser M and Heidtmann HH: A leucine-to-proline substitution causes a defective alpha 1-antichymotrypsin allele associated with familial obstructive lung disease. Genomics 17: 740-743, 1993.

23. Ishii T, Matsuse T, Teramoto S, Matsui H, Hosoi T, Fukuchi Y and Ouchi Y: Association between alpha-1-antichymotrypsin polymorphism and susceptibility to chronic obstructive pulmonary disease. Eur J Clin Invest 30: 543-548, 2000.

24. Turner GA: Haptoglobin. A potential reporter molecule for glycosylation changes in disease. Adv Exp Med Biol 376: 231-238, 1995.

25. Dowling P, O'Driscoll L, Meleady P, Henry M, Roy S, Ballot J, Moriarty M, Crown J and Clynes M: 2-D difference gel electrophoresis of the lung squamous cell carcinoma versus normal sera demonstrates consistent alterations in the levels of ten specific proteins. Electrophoresis 28: 4302-4310, 2007.

26. Bharti A, Ma PC, Maulik G, Singh R, Khan E, Skarin AT and Salgia R: Haptoglobin alpha-subunit and hepatocyte growth factor can potentially serve as serum tumor biomarkers in small cell lung cancer. Anticancer Res 24: 1031-1038, 2004.

27. Hoagland LF IV, Campa MJ, Gottlin EB, Herndon JE II and Patz EF Jr: Haptoglobin and posttranslational glycan-modified derivatives as serum biomarkers for the diagnosis of nonsmall cell lung cancer. Cancer 110: 2260-2268, 2007.

28. Takeda Y, Shinzaki S, Okudo K, Moriwaki K, Murata K and Miyoshi E: Fucosylated haptoglobin is a novel type of cancer biomarker linked to the prognosis after an peration in colorectal cancer. Cancer 15 118: 3036-3043, 2012.

29. Wang B, He YJ, Tian YX, Yang RN, Zhu YR and Qiu H: Clinical utility of haptoglobin in combination with CEA, NSE and CYFRA21-1 for diagnosis of lung cancer. Asian Pac J Cancer Prev 15: 9611-9614, 2014. 